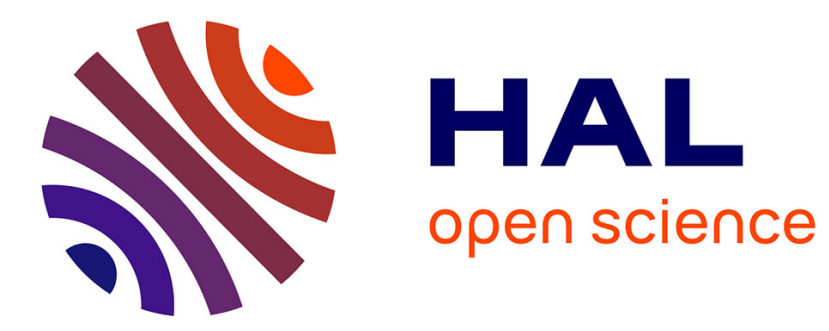

\title{
Quand les mots sortent du cadre ou Ben au supermarché
} Martine Azam

\section{To cite this version:}

Martine Azam. Quand les mots sortent du cadre ou Ben au supermarché. Sociologie de l'art, 2009, Les mondes de l'interprétation, 14 (1), pp.91-114. hal-01252188

\section{HAL Id: hal-01252188 \\ https://hal-univ-tlse2.archives-ouvertes.fr/hal-01252188}

Submitted on 7 Jan 2016

HAL is a multi-disciplinary open access archive for the deposit and dissemination of scientific research documents, whether they are published or not. The documents may come from teaching and research institutions in France or abroad, or from public or private research centers.
L'archive ouverte pluridisciplinaire $\mathbf{H A L}$, est destinée au dépôt et à la diffusion de documents scientifiques de niveau recherche, publiés ou non, émanant des établissements d'enseignement et de recherche français ou étrangers, des laboratoires publics ou privés. 
Azam M. "Quand les mots sortent du cadre ou Ben au supermarché", L'interprétation,

Sociologie de l'art, $\mathrm{n}^{\circ} 14$, Septembre 2009.

\section{Quand les mots sortent du cadre ou Ben au supermarché}

\section{Résumé}

La question de l'interprétation des ouvres trouve un relief particulier lorsqu'elles sortent du cadre où elles sont supposées être vues et, à ce titre, les "objets Ben" destinés aux adolescents offrent une illustration exemplaire de cette convergence entre la logique du marketing et celle d'une problématique artistique. Les étapes de la découverte de Ben, ainsi que les prises dont les adolescents se saisissent pour l'intégrer à leur univers culturel conduisent à interroger la thèse actuelle d'un bouleversement des pratiques culturelles et des échelles de légitimité. Les résultats de l'enquête ne permettent pas de trancher mais ils mettent par contre bien en évidence ce que l'on peut considérer comme un préalable à un réel bouleversement des pratiques culturelles, la migration de l'art dans la sphère du quotidien. En portant l'attention sur ce que les adolescents disent des objets Ben, nous verrons que, si les objets quotidiens permettent la dissémination de l'œuvre en direction de publics élargis, l'inscription de l'art dans le registre du marketing ne conduit pas à sa dilution, pas plus qu'à sa dissolution.

Mots clefs : légitimité culturelle, pratiques culturelles, adolescents, interprétation, limites de l'œuvre, objets quotidiens, Ben, blog

\section{Abstract}

The issue of works interpretation finds a characteristic relief when the works are settled out of their supposed limits. The "Ben things 》, intended for teenagers, offer an exemplary illustration of this meeting between marketing and artistic logics. The stages of Ben's discovering by teenagers as well as the ways the teenagers used Ben's works and integrate it in their cultural world lead to questioning the current thesis of a disruption in cultural uses and legitimacy hierarchies. The enquiry results highlight what we can consider as a prerequisite for a real disruption of cultural practices, migration of art in the daily sphere. In paying attention to what teenagers say about "Ben things" we will see that daily things allow the work to be scattered towards expanding audience. The art entering the marketing register don't lead to his dilution -which supposes a weakening of art content - no more than his dissolution

Keywords: cultural legitimacy, cultural uses, teenagers, interpretation, art work's limits, daily things, Ben, blog.

Resumen

El problema de la interpretación de las obras de arte encuentra un relieve particular cuando ellas salen del contexto de donde se supone que debieran ser vistas y a este respecto, "los trabajos de Ben" que se destinan a los adolescentes ofrecen una ilustración ejemplar del punto de intersección entre la lógica de marketing y aquella de la problemática artística. Las etapas en el descubrimiento de Ben, y la manera que los adolescentes adoptan para integrar su universo cultural llevan a interrogar la tesis actual de una transformación radical de prácticas culturales y de escalas de legitimidad. Si los resultados de la encuesta no permiten de decidir, estos resultados, en cambio, ponen en evidencia lo que se puede considerar como un preliminar a una verdadera transformación de prácticas culturales, la migración del arte en las esferas del diario vivir. Prestando un cuidado a lo que los adolescentes dicen de los trabajos de Ben, veremos que si los objetos cotidianos permiten la diseminación de la obra en dirección de un público amplio, la inscripción del arte en el registro del marketing no conduce a su dilución - que supone un debilitamiento del contenido del arte - ni a su disolución.

Mots clefs : legitimidad cultural, practicas culturales, interpretación, limites de la obra de arte, objetos cotidianos, blog. 
Les questions que l'art contemporain pose à la sociologie de la réception sont inscrites au cœur de pratiques artistiques qui n'ont cessé d'interroger la notion même d'art. De surcroît, les modes de transmission et de diffusion des œuvres et les usages médiatico-publicitaires dont l'art fait l'objet ont favorisé une relation occasionnelle ou accidentelle avec les choses de l'art. Ces deux éléments rendent alors tout à fait aigüe la question de l'interprétation des œuvres lorsqu'elles sortent du cadre où elles sont supposées être vues. Le travail de Benjamin Vautier offre une illustration exemplaire de cette pénétration de la sphère du quotidien par l'art.

Plus connu sous le nom de Ben, cet artiste qui fut l'un des promoteurs du mouvement Fluxus en France, a choisi dès les années 50 de déborder au sens propre comme au sens figuré du cadre du monde de l'art. Il se situe dans la filiation de Duchamp et des dadaistes, pour lesquels l'art est d'abord l'idée. Si cette obligation d'innovation s'inscrit dans la tradition de rupture et de dépassement inhérente à la notion même de contemporanéité, Ben l'a développé de façon radicale pour ne plus garder de l'œuvre que l'idée. Dans cette logique du plus court chemin, il devient inutile de réaliser l'œuvre elle-même puisque la vérité de l'œuvre se trouve dans ce qui précède sa mise en forme, et rien n'est plus direct et plus simple que de recourir aux mots pour exprimer l'idée. Les mots et les phrases deviennent alors la matière première de l'œuvre ; portés par une graphie singulière, ils ont très vite caractérisés un large pan de son travail artistique.

Dans le travail de Ben, la portée des mots qui véhiculent l'idée est d'autant plus forte qu'elle n'est pas distraite par une mise en scène esthétisante. La graphie ronde sur fond noir qu'il utilise de façon habituelle s'identifie très facilement, tout comme sa signature. La logique dans laquelle cet artiste a placé son œuvre combinée avec l'identité graphique qu'il a adoptée ont permis sa notoriété au-delà des arènes confidentielles du monde de l'art contemporain, l'amenant à une reconnaissance très large, explicitement visée par l'artiste : "Tout le monde doit pouvoir dire : c'est du Ben". Sollicité par des grandes marques désormais soucieuses de vernis culturel, Ben est partout et fait partie de ces rares artistes contemporains connus par le grand public qui ne connait et/ou ne retient de lui que cet aspect de son travail.

Par ses éléments - l'idée comme œuvre, les mots comme matériaux, une identité graphique forte - le travail de Ben portait en lui cette possible extension hors du champ artistique. En effet, si l'idée d'œuvre suffit, peu importe alors le support qui la véhicule : ce peut être une toile, mais ce peut être également une trousse, un stylo ou un tee-shirt..., c'est à dire des supports appartenant à l'univers quotidien et reproductibles industriellement.

D'abord déclinés sur les objets et accessoires vendus dans les galeries et boutiques des musées, puis dans des boutiques de gadgets et cadeaux, les phrases et les mots de Ben ont envahi, au milieu des années 2000, les rayons papeterie de la rentrée dans les supermarchés au travers de produits plus particulièrement destinés aux adolescents. La prolifération des phrases de Ben sur des produits de consommation courante conduit à se demander quel sens donner à cette "sortie de cadre" et à s'interroger sur ce qu'il reste de la nature artistique du travail de Ben. Les réponses pourraient être cherchées du côté de ce que dit l'artiste sur son travail, ainsi que du côté de ce que disent les spécialistes - critiques, historiens d'art, philosophes - mais notre objectif est autre : il est de donner la parole de ceux à qui sont destinés ces objets et, parmi eux, à ceux qui se sentent concernés par ces objets : les adolescents.

Cet article se propose de poursuivre une réflexion engagée sur les modalités expérientielles de la rencontre avec les œuvres. Un premier temps, centré sur la notion de prise, avait permis de pointer les compétences perceptuelles, cognitives et discursives déployées par les récepteurs ${ }^{1}$. Un second temps avait montré que le cadre dans lequel s'inscrit la rencontre avec l'œuvre, au sens de contexte physique et de segment d'activité, peut faire basculer l'identification de l'objet et par suite son interprétation ${ }^{2}$. Les deux volets de ce travail avaient ainsi mis en évidence la façon dont les prises offertes par les œuvres, d'une part, et les prises offertes par le cadre de l'activité, d'autre part, organisent la relation à l'art et l'interprétation des œuvres. En portant l'attention sur ce que les adolescents disent des objets Ben, nous voulons enrichir cette réflexion d'un nouvel élément : la question de la nature de l'objet.

En effet, nous avons jusqu'ici traité de la relation à l'œuvre, entendue comme objet unique, mais que devient la relation à l'art quand celui-ci migre sur des objets quotidiens ? Après W. Benjamin, l'idée s'est imposée d'une

\footnotetext{
${ }^{1}$ M. Azam, "La rencontre avec le Musée Khombol", Vers une sociologie des æeuvres, sld Majastre O. et Pessin A., L'Harmattan, Collection Logiques sociales, 2001.

${ }^{2}$ M. Azam, "La pluralité des rapports à l'art. Etre plus ou moins public", Les non-publics. Les Arts en réception, sld Ancel P. et Pessin A., L'Harmattan, Collection Logiques sociales, 2004.
} 


\section{Toulouse}

sorte de dégressivité de la nature artistique de l'objet selon une graduation allant de l'œuvre originale à sa reproduction. En perdant son aura, l'œuvre reproduite perdrait également en légitimité. Depuis le milieu des années 80 , des artistes se sont appliqués à contrecarrer cette conception en produisant des œuvres démultipliées, des $2 u v r e s$ ouvertes qu'il n'est plus possible de délimiter ${ }^{3}$. A leur façon, les objets Ben contribuent à cette remise en cause des limites de l'œuvre en même temps qu'à celle des échelles de légitimité en art. C'est en nous appuyant sur l'interprétation que les adolescents en font que nous chercherons à alimenter cette réflexion.

Le questionnement s'organise en deux temps. Le premier nous introduit dans l'univers des adolescents et l'on cherche à cerner la façon dont opèrent sur eux ces mots et ses phrases qui sont au principe du travail de Ben. Au travers quelques résultats de l'enquête, on tente de restituer le parcours qui mène de la découverte des objets Ben à la connaissance de l'artiste et de repérer les "principes actifs" dont se saisissent les adolescents.

Quelle est pour les adolescents la nature exacte de ces objets ? Sont-ils encore de l'art ou doit-on les ranger au rayon des produits dérivés ? Les adolescents distinguent-ils un Ben-artiste et un Ben-homme de marketing ? Ces questions ouvrent sur le second temps de la réflexion qui s'intéresse à la nature et la place de ces objets dans les référents culturels des adolescents. Les changements mais également des ambiguïtés dont ils sont porteurs interrogent plus largement la thèse actuelle d'un bouleversement des pratiques culturelles.

\section{1- Le rapport à l'œuvre}

Dans la perspective de traiter de la relation que les adolescents entretiennent avec le travail de Ben, les blogs constituent un matériau précieux puisque s'y exprime une communauté d'intérêts. Après exploration de la toile, trois blogs ont retenu notre attention ${ }^{4}$ : faits par des internautes, entièrement dédiés à l'œuvre de Ben, ils sont tout à fait révélateurs de la relation que ces amateurs entretiennent avec l'œuvre et avec l'artiste et les échanges entre internautes permettent d'observer ce qui fait sens pour eux. Initialement envisagés au titre de la phase exploratoire, ils se sont révélés d'une telle richesse qu'ils ont constitué le matériau principal de l'enquête ${ }^{5}$. Des entretiens ${ }^{6}$ ont permis d'approfondir certains aspects de l'analyse.

Chaque blog est constitué de sections présentant une image d'une œuvre et laissant la possibilité d'écrire des commentaires. Les images proposées sont des photos de pages d'agendas Ben, des photos de toiles, d'installations ou de performances... Le blog le plus important en nombre d'œuvres présentées, de communications laissées, de visiteurs identifiables, est également celui présentant exclusivement des œuvres distinctes du répertoire produit à destination des objets de grande consommation. De la sorte, il permet d'accéder à d'autres aspects de l'œuvre que ceux connus du grand public.

L'amateur de Ben est une amatrice des proportions écrasantes : sur les 248 visiteurs, on a identifié $76 \%$ de filles et $8 \%$ de garçons, qui pour l'essentiel ont entre 11 et $15 \mathrm{ans}^{7}$. Cette prédominance des filles peut être rapprochée du rapport spécifique des adolescentes à l'écriture -comme en atteste la pratique du journal intime dont le blog en est une déclinaison moderne- et l'on peut comprendre qu'une œuvre utilisant les mots trouve chez elles un écho particulier ${ }^{8}$.

\section{Les étapes de la découverte}

Le moment décisif dans la découverte de l'oeuvre de Ben semble être lié à l'acquisition d'un objet -trousse, agenda, stylos sont les articles les plus cités- mais il apparaît que cette étape est précédée d'une familiarité diffuse de l'adolescent avec ces phrases blanches sur fond noir. De même, dans les cas où l'adolescent a découvert un blog dédié par hasard, il en entreprend la visite car le nom de Ben lui est déjà connu.

\footnotetext{
${ }^{3}$ O Delleaux, Les multiples et "autres" multiples depuis le milieu des années 80? Enjeux et mutations, thèse d'Histoire de l'art contemporain, Université de Rennes 2, mars 2006.

${ }^{4}$ Fréquenté quasi exclusivement par des personnes qui manifestement se connaissent pour appartenir au milieu de l'art et qui poursuivent leur polémique sur la toile, le blog de l'artiste a été écarté de l'analyse.

${ }^{5}$ Réalisé par des jeunes filles de 17 à 18 ans dont l'objectif est de faire connaître Ben, ces trois blogs totalisent 1591 messages, appelés "com" par les internautes

${ }^{6}$ Seize entretiens auprès de lycéens et collégiens qui possèdent des objets Ben avaient pour objectif d'éclairer les raisons qui ont présidé au choix des objets et, plus largement, les circonstances de la découverte de l'œuvre de Ben. Ils ont également permis de préciser la place de ces objets dans l'univers culturel de jeunes.

${ }^{7}$ Bien qu'il ne soit pas toujours possible de connaître l'âge exact de ces internautes, il s'avère que cette population est très jeune (moins de 15 ans) et féminine.

${ }^{8} \mathrm{Ce}$ constat rejoint celui de S. Octobre remarquant que les filles échangent plus que les garçons sur leurs passions, leurs sentiments, leurs goûts et que ces échanges sont d'abord pour elles l'occasion de mettre à l'épreuve leurs catégories cognitives et affectives.
} 
La seconde étape, l'exploration de la toile, correspond à la collecte "d'images-Ben" et à ce titre, ces blogs constituent des ressources d'illustrations destinées à être imprimées ou importées. Mais, au-delà de la collecte elle-même, il y a, dans la systématicité avec laquelle les adolescents parcourent toutes les images et dans leur façon de réagir à certaines d'entre elles, la recherche d'une ré expérimentation du plaisir initial produit par les mots. Cette ré expérimentation est aisément satisfaite sur le net où l'accès aux phrases est gratuit et facile, de même qu'elle peut également s'effectuer par la manipulation des objets dans les magasins, ainsi que le révèlent les entretiens.

L'enchaînement de ces deux étapes se donnent à voir très clairement, mais il n'en est pas de même pour deux autres moments dont l'articulation au processus de découverte plus incertaine. En effet, si l'on pense ce goût pour Ben sur le modèle d'une progression dans la connaissance d'une œuvre, la $3^{\mathrm{e}}$ étape serait celle de la visite du site de l'artiste et la $4{ }^{\text {ème }}$ serait la découverte physique des œuvres dans des lieux d'art.

De façon surprenante, ils sont peu nombreux à visiter le site de l'artiste, et quand ils l'ont fait, ils se montrent très déroutés par sa présentation foisonnante et le quittent très vite. La visite du blog de l'artiste révèle de même que les adolescents ne cherchent pas à entrer en communication avec lui en dépit de l'enthousiasme manifesté ailleurs pour son travail. Plus à l'aise sur les sites dédiés, ils préfèrent reprendre leur chasse aux phrases et leurs communications avec les pairs. Ils retrouvent là un terrain connu puisque tous les blogs sont identiques dans leur structure en raison du formatage imposé par Skyblog-

La découverte des œuvres originales de cet artiste dans un lieu d'art semblait devoir constituer l'aboutissement dans un parcours de découverte d'une oeuvre. Même si les données manquent pour le confirmer ${ }^{9}$. rien ne permet de savoir si la rencontre avec les œuvres originales dans un lieu d'art a précédé ou suivi la connaissance de l'œuvre que procure la fréquentation de la toile, et les deux cas sont présents :

Lorsque des témoignages font référence à une découverte de l'œuvre en situation, c'est l'occasion pour les visiteurs d'exprimer un degré supérieur de connivence avec l'œuvre et l'artiste et il arrive souvent qu'un internaute apporte un complément d'information. La connaissance de l'œuvre de l'artiste trouve également à se manifester autour de l'interprétation qu'il convient de donner à une œuvre : parmi les œuvres mises en ligne, la photographie d'un objet dont la forme interroge et qui porte la mention "Esclave" :

\author{
-C'est un bracelet?? \\ -je pense pas \\ -c'est un cendrier. On est esclave du cendrier et de la clope je pense, non? \\ -c'est un bracelet! mais ça coûte très cher!! je suis tombée sur le site et le bracelet vaut 3000 euros !
}

\title{
Un principe actif : Ego en miroir ?
}

Les phrases de Ben figurant dans les blogs dédiés peuvent être classées de la façon suivante : des états possibles de égo ("Seul"), des injonctions sur des choses à faire ("N'oubliez pas de tomber amoureux"), des façons d'être ("Etre naturel"), des réflexions sur l'état du monde contemporain ("Bientôt il n'y aura plus de place"), sur l'art en général ("Art doesn't exist") et sur Ben-artiste en particulier ("J'ai voulu abandonner l'art mais j'en ai fait, de l'art"). Il existe également des phrases déclaratives qui engagent ("Je suis pour...").

Les phrases et les mots sont ce par quoi les adolescents entrent dans l'œuvre de Ben et l'artiste ayant fait de "égo" une partie importante de son travail, on ne sera pas surpris que ces phrases retiennent l'attention des adolescents en nourrissant leur regard narcissique, ils se reconnaissent en elles. Ces phrases peuvent être choisies parce que l'adolescent se reconnaît en eux :

"... y avait plusieurs phrases... j'ai pris celle là parce ce que ça me correspondait : j'avais de l'ambition, je voulais réussir mon année"

Elles peuvent également cristalliser quelque chose de diffus ; dans ce cas, elles agissent sur l'adolescent comme le révélateur d'un possible dans lequel il se projette. En se saisissant de la phrase, il la fait sienne et peut présenter aux autres un aspect de ce qu'il se sent être, les phrases sont alors des "vitrines de soi" :

"'Réussir ma vie, mon bac, mes amours... C'est aussi montrer aux autres qu'on est une personne bien, qui est valable puisqu'on veut faire des choses."

\footnotetext{
${ }^{9}$ Le nombre visiteurs faisant allusion à des expositions est trop faible et les entretiens n'ont pas permis d'éclaircir ce point, aucun des adolescents interrogés n'ayant eu l'occasion de voir des œuvres originales.
} 
D'autres mots amplifient un sentiment, un désir : c'est le cas de "Libre" que les adolescents prennent à la fois comme un souhait et un objectif. À la lecture des commentaires s'impose l'idée, non pas d'une identification entre les adolescents et l'artiste, mais d'une projection comme l'indique très bien cette jeune fille :

\section{" j'ai l'impression qu'on me tendait la main (....) que c'était moi qui l'avait écrit»}

Et si cette projection est possible, c'est en raison de l'interprétation très ouverte que permettent ces phrases. Ainsi, à un visiteur regrettant que le blog ne propose pas d'interprétation des œuvres de Ben, un échange s'en est suivi entre la créatrice du blog et les participants, indiquant clairement que le "moi" est en même temps le "chacun de nous" :

\section{-" l'interprétation que j'ai des images de Ben sont assez perso dans le sens où chaque phrase est un bout de vie"}

-"chaque phrase parle différemment pour chaque personne et c'est ça qui est bien...."

Doit-on voir derrière les réactions à ces œuvres une contamination généralisée de l'art par l'extime ${ }^{10}$, si caractéristique des derniers développements de la culture médiatique ? La première réserve à cette hypothèse tient à la nature même du travail de Ben. Même si l'artiste "dissèque un égo qu'il s'est donné pour fondement" ${ }^{11}$ les registres qu'il emploie sont différents des problématiques artistiques contemporaines - si nombreuses - jouant sur le pathos et déployant des procédés destinés à provoquer l'émotion de l'interlocuteur. Les œuvres-phrases de Ben, y compris maintiennent une distance produite autant par leur présentation quasi uniforme que par l'humour et l'autodérision dont elles sont porteuses ${ }^{12}$. La seconde réserve tient au volume des réactions suscitées : ce sont les considérations sur la vie ou le monde qui suscitent le plus d'échanges de la part des adolescents. Ainsi parmi les images les plus commentées, on trouve les phrases "Vous avez tous peur", "Art doesn't exist" et "Rire est une arme, on peut mourir de rire"; la photo de Ben accrochant la banderole "J'ai mangé un ouf dur hier à 19h30", cette dernière image en déclenchant des phrases d'adolescents dans la même veine. Autre exemple, le mot "Libre" inspire les réflexions des internautes et les discussions qui s'engagent sur le blog sont l'occasion de commenter le thème général soulevé par l'œuvre et de discuter de sa déclinaison à l'adolescence.

\section{2- La nature et la place de ces objets}

Les analyses extraites du dépouillement des blogs indiquent les prises utilisées par les adolescents pour s'approprier certains aspects de l'œuvre de Ben. Mais qu'ont-ils perçus exactement dans ces objets ? Font-ils une différence entre les produits de grande consommation signés Ben et d'autres articles griffés ? La découverte d'autres aspects de l'œuvre de Ben les conduit-elle à réinstaurer à la traditionnelle partition entre l'œuvre originale et ses multiples, les produits dérivés?

\section{Ben plus fort que Nike}

De graphie quasi-scolaire, très courte et facile à retenir, les phrases figurant sur les objets traitent de thèmes proches des préoccupations de ce public. Pour ces raisons, les textes sont très rapidement perçus comme appartenant à un ensemble. Même dans les cas - très rares ${ }^{13}$ - où le jeune ne sait pas que Ben est un artiste, il ne fait pas de doute pour lui que l'auteur de ces lignes est une personnalité et non une marque. Ce qui leur permet de passer de l'individualité à l'artiste n'apparaît pas vraiment, la réponse s'imposant comme une évidence : "Tout le monde le sait !". Toutefois, au-delà du cas de Ben dont la médiatisation est importante, les adolescents définissent l'artiste comme celui qui fait des choses auxquelles les autres ne pensent pas et qui les signe. Savoir dans quelle catégorie classer cet artiste importe peu pour eux : que Ben expose dans des galeries ou des musées ainsi qu'ils le découvrent rapidement sur la toile par les sites associés, est un "plus" dans la mesure où cela confirme le bien-fondé de l'intérêt qu'ils lui portent, mais cela n'influe pas fondamentalement sur l'idée qu'ils s'en font: "Ben c'est quelqu'un!" "Ben c'est un grand artiste".

En suivant leur logique, on peut comprendre que la dimension artistique de l'objet prime sur la fonctionnalité : en achetant un objet Ben, les jeunes achètent avant tout ce qui est pour eux un objet d'art. Les discours peuvent être synthétisés autour de ces deux propositions articulées : il s'agit là d'objets qui ont une utilité et qui sont produits en masse et ce titre ils ne sont pas exactement des objets d'art; pour autant, ces objets ce ne sont pas des objets

\footnotetext{
${ }^{10}$ Il faut entendre par là : l'intime exposé, terme suggéré par Jean-Pierre Rouch.

${ }^{11}$ Hervé Bize, Ben portrait d'un artiste carnivore, Site de l'artiste

${ }^{12}$ Cette caractéristique si appréciée par les jeunes prend sa source dans les composantes des deux mouvements dont Ben se revendique : le dadaïsme et ses jeux sur l'absurde et dans les performances de Fluxus.

${ }^{13}$ Un seul cas s'est présenté dans le cadre de cette enquête, entretiens et blogs compris
} 
Martine Azam,

LISST-CERS

Toulouse

comme les autres car ils sont "beaux". Autrement dit, l'absence des critères d'unicité et de finalité sans fin ne suffit pas à les disqualifier en tant qu'objet d'art : ces objets sont de l'art parce qu'ils satisfont au critère de beauté! L'idée de la beauté des objets Ben est récurrente mais si l'on pousse cette question au-delà des premières déclarations, il apparaît que la beauté dont parlent les adolescents renvoie moins à la forme de l'objet qu'elle ne se niche dans l'idée proposée par l'artiste, dans la réflexion ouverte à l'adolescent et dans l'émotion que cette ouverture lui procure. La beauté se situe dans l'état cognitif déclenché par la phrase et l'émotion dont parle les adolescents est celle procurée par le questionnement qui soudain s'ouvre à eux : elle se trouve dans l'expérience même de la rencontre avec l'œuvre.

\section{Ben aussi fort que Bob Sinclar}

L'univers culturel des adolescents est composé de références leur permettant de se reconnaître et de se distinguer les uns des autres. Si de nombreuses études ont montré la prééminence de la musique et du cinéma dans leurs choix culturels, on peut se demander quelle place occupe Ben dans cet ensemble : y a t-il là un effet de distinction différent de celui procuré par d'autres ancrages, comme celui de la musique rock par exemple ? Il faut être prudent en raison d'un terrain qui demande à être approfondi, mais il semble qu'aux yeux des adolescents rencontrés, l'important est que Ben soit artiste. Artiste, acteur, musicien, la question d'une hiérarchie entre ces divers mondes de l'art est une question d'adulte. Plus encore, si on les interroge frontalement sur ce sujet, ils expriment une opposition de principe à toute hiérarchie entre les disciplines. Dans leur univers, Ben est aussi important que Bob Sinclar, les Red Hot Chili Pepper, ou Johnny Depp ; aussi important encore que le concepteur de l'IPOD. Toutes ces figures participent à un ensemble de références culturelles et artistiques dont ils saisissent les différences ${ }^{14}$ mais qu'ils n'éprouvent pas le besoin de hiérarchiser.

Une autre idée forte s'impose dans les discours des adolescents : l'art peut se nicher partout et peut se répandre par le biais des objets. Présente chez ceux qui, par leur milieu social, connaissent l'existence d'une hiérarchie dans les œuvres de culture, cette idée l'est également chez les adolescents de milieux populaires que nous avons rencontrés. Pour tous, et de façon assez tranchée, il existe d'une part le monde des adultes - associé à la culture légitime, aux musées, à l'école, aux artistes "vieux ou morts" - et d'autre part, la culture des jeunes - associée à une culture fluide, vivante et ouverte. L'art des musées s'oppose à l'art pour tous, au double sens de :

- l'art fait par tous : tout le monde peut être artiste d'une certaine façon et Ziddane est un artiste du ballon

- l'art à destination de tous : l'art qui se diffuse sur tous les médiums et auquel tout le monde peut avoir accès ; à ce titre, Ben sur les trousses en est le parfait exemple.

Dans cette logique d'un art pour tous, l'œuvre n'est plus unique puisque démultipliée à l'envi sur les objets, c'est l'artiste qui désormais est porteur de cette unicité. L'aura n'est plus attachée à l'œuvre, elle s'est déplacée sur la personne de l'artiste pour s'étendre à tous les objets qu'il créé, qu'il s'agisse d'œuvres originales, de multiples numérotés ou non, ou d'objets industriels. Les livres, les disques, tout comme les objets Ben, sont pour eux de la culture. Dans les discours apparaît ainsi le glissement qu'ils opèrent de l'art vers la culture : l'un et l'autre ne sont pas de nature différente, ni séparés par une frontière, mais ils se situent dans un continuum.

Alors, tout se vaut-il pour les adolescents ? Comment interpréter cette indifférenciation: doit-on y voir la manifestation d'un "manque de culture" ${ }^{15}$, d'une adhésion à un relativisme ambiant ou est-ce là le signe d'une remise en question de la légitimité culturelle ...?

\section{Une remise en question de la théorie de la légitimité culturelle ?}

La disgrâce qu'enregistre le recul des pratiques cultivées a déjà été mise en évidence dans plusieurs enquêtes ; les lectures qu'en donnent les spécialistes, bien que comportant de sérieuses nuances, s'organisent autour de la trame suivante.

Un des effets de l'économie médiatico-publicitaire est d'avoir imposé de nouvelles voies de consécration sociale et culturelle. En épousant les formes spécifiques de cette économie, l'art est désormais noyé dans une sorte de grand-tout culturel et communicationnel où règne l'indistinction entre les registres et les genres. La période de règne médiatique que nous vivons est celle de la mise à mal de l'opposition entre culture cultivée et culture de masse. L'émergence de nouvelles pratiques culturelles bouscule autant l'échelle de légitimité culturelle issue de

14 "Quand je suis allée sur le site, j'ai vu un homme avec les cheveux blancs, un peu dans le genre de Picasso" : cette association est spontanément mobilisée par cette jeune fille et indique qu'existe de façon distincte une catégorie "artiste peintre" qui n'est pas amalgamée à celle des musiciens par exemple.

15 Au sens de "culture cultivée" 
la modernité que le cadre théorique conçu pour rendre compte de la correspondance entre catégorie sociale supérieure et goûts cultivés, c'est-à-dire la théorie de la légitimité.

Dans cet ordre d'idées, les études anglo-saxonnes ${ }^{16}$ pointent l'existence de nouveaux types de rapport à la culture qui opposent les omnivores aux univores, indiquant que l'on assiste actuellement à une transformation des règles de la distinction. Les changements observés sur les populations adultes semblent confirmés par les études sur les adolescents dont les comportements sont le signe d'une accélération et d'une amplification de ce mouvement dans les nouvelles générations. Les adolescents sont un groupe-repère pour deux raisons : leur opposition au monde adulte les conduit à développer une culture spécifique et ils sont la première génération à avoir grandi dans un environnement médiatique très riche combinant bouleversements audiovisuels et explosion de l'informatique domestique. Ces spécificités les ont conduits à établir leur propre échelle de valeur en matière de référents culturels. De façon plus prononcée que dans les autres, ce groupe enregistre des changements importants dans le rapport aux œuvres avec un recul de la culture consacrée, associé à une valorisation de l'éclectisme et à une certaine forme d'anti-intellectualisme.

Si les observations tirées de cette enquête semblent conforter cette thèse et montrer l'existence d'un relativisme culturel qui prend la forme particulière d'un rejet de la culture légitime, le cas étudié ici montre que cette remise en question est d'abord celle des hiérarchies que les adolescents associent au monde adulte ; elle n'est qu'incidemment ou conséquemment la remise en question des expressions artistiques qui en émanent. On pourrait ainsi suggérer que, au delà des facteurs combinés liés à une mobilité généralisée ${ }^{17}$, la remise en cause de la légitimité culturelle est en réalité celle de l'univers adulte par les générations montantes en tant que groupe social. Dans la période contemporaine, cette remise en cause trouverait à s'ancrer durablement dans la fusion des univers dans lesquels les jeunes ont grandi : l'univers médiatico-publicitaire et celui des technologies de l'information et de la communication. Métaphoriquement, on pourrait dire que le "Mai 68" des adolescents d'aujourd'hui passe par leur puissance connectée et consommatoire qui tend à opposer à l'inertie d'une société vieillissante des goûts et des pratiques culturelles s'organisant, non plus sur le modèle de diffusion descendant mais un modèle de diffusion horizontal, ascendant et rhizomatique.

Toutefois, cette lecture -la remise en question des hiérarchies culturelles traditionnelles et le prolongement prospectif qui en découle- nous semble devoir être tempérée en raison, sinon des contradictions du moins des ambiguïtés que nous avons observé. Il apparaît notamment que la signature reste un élément fort pour permettre l'identification de l'artiste, et, plus surprenant, que la signature conserve sa dimension d'authentification. En témoigne cet échange :

-"c'est une imitation de Ben Vautier mais ce n'est pas lui car je ne sais pas si vous avez remarqué mais lui il signe toujours !!"

-"si si, mais l'image est p-ê coupée ou alors c'est effectivement pas de lui "

Un second élément vient rendre moins nette l'interprétation d'une remise en question de la légitimité culturelle : si nous avons vu que dans l'esprit des adolescents l'aura de l'œuvre originale se démultiplie à proportion du nombre d'objets empruntés par les mots, on retrouve tout de même chez certains, exprimée au détour d'une phrase, l'envie suscitée par un original hors de portée. Ainsi, d'un côté il n'y a plus de limites à l'œuvre puisque la trousse Ben est un objet d'art, mais d'un autre coté l'œuvre garde un noyau : l'original.

Ces deux éléments -l'importance de la signature pour authentifier et le désir que suscite encore l'original- doivent inciter à la prudence. Le renversement des critères classiques en art n'est pas complet et l'on peut se demander si ne subsiste pas encore, de façon non dite, la distinction entre "la vraie œuvre d'art" et ses multiples industriels, et cela en dépit du déni des adolescents.

Pour résumer donc, d'un côté la thèse de la légitimité culturelle qui vacille sous l'effet du constat d'un changement dans les pratiques, constat conduisant à penser que le modèle omnivore/univore organise selon de nouveaux principes la distinction des goûts et des pratiques. De l'autre, des indices fournis par cette enquête qui, tout en confirmant ce relativisme culturel assorti du rejet de la hiérarchisation montrent la persistance des critères classiques comme l'original et la signature. Sommes-nous en présence d'une superposition de deux ordres de légitimité dont on peut penser qu'ils vont durer dans le temps? S'agit-il du reliquat d'un rapport à la culture sur le

\footnotetext{
${ }^{16}$ A ce sujet, cf les travaux de l'américain Peterson R.A et notamment, "Le passage à des goûts omnivores : notions, faits et perspectives", Sociologie et Sociétés, Vol 36, n¹, 2004.

${ }^{17}$ Pour Peterson R.A., cette mobilité généralisée impose le "pattern de l'omnivorité". Cette mobilité est de plusieurs ordres : circulation physique des personnes entre les différentes zones du globe et du territoire, circulation des idées par leur supports et les moyens ce communications, mobilité sociale forte qui est aussi une forme de circulation.
} 


\section{Toulouse}

déclin, sorte d'écho amené à s'estomper avant de disparaître ? À l'évidence, cette rapide discussion à partir des éléments fournis par notre enquête ne permet pas de trancher en faveur d'une de ces interprétations ${ }^{18}$.

Sur le terrain de l'interprétation de l'œuvre, il reste pourtant un élément qui doit être souligné de façon ferme. A la question d'une remise en question des limites de l'œuvre, et sans avoir connaissance des explicitations dont un artiste accompagne toujours sa démarche, les adolescents ont clairement répondu : les objets Ben sont une extension de l'œuvre. Que cette extension se fasse en utilisant les voies du marketing n'enlève rien, ni à l'œuvre ni à l'artiste. En "sortant du cadre", Ben a rencontré les consommations adolescentes : c'est bien parce que les deux parties se sont retrouvées hors du cadre du monde de l'art que cette rencontre est devenue possible et que les adolescents ont pu entrer dans univers artistique singulier. Qu'elle qu'en soit la durée ${ }^{19}$, cette sortie de cadre a ouvert une fenêtre supplémentaire dans l'univers culturel adolescent -celle des arts plastiques contemporains-.

Les mots de Ben sont de la valeur ajoutée -au sens propre comme au figuré ; valeur ajoutée à la trousse, valeur ajoutée à eux-mêmes. En circulant sur les objets, l'art se propage, sans toutefois se diluer ; il se dissémine sans toutefois se dissoudre. Ben "utilise le système" mais cela ne remet pas en cause sa légitimité à être artiste : très pragmatiquement, les adolescents considèrent que l'artiste se sert de ce qui est à sa disposition. Les mots sont sortis du cadre pour migrer sur les trousses et les stylos mais Ben au supermarché est toujours un artiste.

\section{BIBLIOGRAPHIE}

Azam M., "La rencontre avec le Musée Khombol", Vers une sociologie des æuvres, L'Harmattan, Collection Logiques sociales, 2001.

Azam M., "La pluralité des rapports à l'art. Etre plus ou moins public", Les non-publics. Les Arts en réception, L'Harmattan, Collection Logiques sociales, 2004.

Bevort E., Bréda I, Les jeunes et Internet : représentations, usages et appropriations, Rapport de Recherche, CLEMI, 2001

Boissière A., La reproductibilité technique chez Walter Benjamin, revue Demeter, Dec. 2003, Université Lille 3 , www.univ-lilli3.fr/revues/demeter.

Delleaux O., Les multiples et "autres" multiples depuis le milieu des années 80 ? Enjeux et mutations, thèse d'Histoire de l'art contemporain, Université de Rennes 2, mars 2006.

Peterson R.A, "Le passage à des goûts omnivores : notions, faits et perspectives", Goûts, pratiques culturelles et inégalités sociales : branchés et exclus, Sociologie et Sociétés, Vol 36, n¹, 2004.

Octobre S., Les loisirs culturels des 6-14 ans, DEPS, Documentation française, 2004

Octobre S., La fabrique sexuée des goûts culturels, construire son identité sexuée de fille ou de garçon à travers les activités culturelles ; Ministère de la culture et de la communication, Développement culturel, $\mathrm{n}^{\circ} 150$, Décembre 2005

\footnotetext{
${ }^{18}$ Et sur le seul aspect empirique, il faudrait que soient conduites des investigations supplémentaires Ainsi, nous ne disposons pas d'éléments suffisants pour dire si les amatrices les plus accomplies de Ben sont issues des catégories sociales favorisées. De même, des investigations devraient éclairer la façon dont les adolescents choisissent et articulent leurs référents culturels, particulièrement en reposant la question de la façon dont ils construisent la distinction entre art et divertissement. Last but not least, le suivi d'une cohorte d'adolescents serait nécessaire pour déterminer si les changements observés (notamment cette absence de hiérarchie entre les genres qu'ils revendiquent et que soulignent les enquêtes) relèvent d'un effet d'âge ou d'un effet de génération.

${ }^{19}$ Les blogs sur Ben ont tous été créés en 2005/2006. Les produits Ben et l'interet qu'il suscite en tant qu'artiste seront-ils frappés d'obsolescence ? En effet, si l'art emprunte les voies du marketing, pourra t-il échapper à la logique de séduction qui s'assortit du renouvellement continu des produits de consommation?
} 
Martine Azam,

LISST-CERS

Toulouse 